\title{
Potential role of pharmacogenomics testing in the setting of enhanced recovery pathways after
}

\section{surgery}

\author{
Hamdy Awad' \\ Ahmed Ahmed ${ }^{2}$ \\ Richard D Urman ${ }^{3}$ \\ Nicoleta Stoicea ${ }^{1,4}$ \\ Sergio D Bergese ${ }^{1,5}$ \\ 'Department of Anesthesiology, The \\ Ohio State University Wexner Medical \\ Center, Columbus, OH, USA; \\ ${ }^{2}$ Department of Anesthesiology, The \\ University of Texas, Houston, TX, USA; \\ ${ }^{3}$ Department of Anesthesiology, \\ Perioperative and Pain Medicine, Brigham \\ and Women's Hospital, Harvard Medical \\ School, Boston, MA, USA; ${ }^{4}$ Department \\ of Biological Chemistry and \\ Pharmacology, The Ohio State University \\ Wexner Medical Center, Columbus, $\mathrm{OH}$, \\ USA; ${ }^{5}$ Department of Neurological \\ Surgery, The Ohio State University \\ Wexner Medical Center, Columbus, \\ $\mathrm{OH}$, USA
}

This article was published in the following Dove Press journal: Pharmacogenomics and Personalized Medicine

\begin{abstract}
In 2001, a group of European academic surgeons created the Enhanced Recovery After Surgery (ERAS) study group and established the first official ERAS protocol. One of the most significant challenges during ERAS implementation is variability of drugs used throughout the perioperative period. Pharmacogenomic testing (blood or saliva) results (obtained within approximately $48 \mathrm{hrs}$ ) provide guidelines on how to prescribe the optimal drug with the optimal dosage to each patient based on an individual's unique genetic profile. Pharmacogenomic testing of various methods of multimodal analgesia is an essential element of ERAS protocols spanning the entire perioperative period to ultimately optimize postoperative pain control. The key goal for anesthetic management in ERAS protocols is to facilitate rapid emergence by using the shortest acting agents available, thus accelerating recovery and reducing length of stay, hospital expenses, and postoperative complications. Postoperative nausea and vomiting (PONV) is an additional challenge that should be overcome to ensure an enhanced recovery and shorter length of stay with the use of antiemetics. Postoperative ileus (POI) can result in longer hospital stay with increasing susceptibility to associated morbidities along with an increase in associated hospitalization costs. Geneticsguided pharmacotherapy and its impact on clinical outcomes should be thoroughly studied for better understanding and managing drug administration in the settings of ERAS.
\end{abstract}

Keywords: pharmacogenomics, perioperative outcome, pharmacogenomics testing, Enhanced Recovery After Surgery

\section{Introduction}

Enhanced Recovery After Surgery (ERAS) is a multidisciplinary perioperative approach that has been developed and adopted over the last twenty years to provide a perioperative value-based care and improvement of the surgical outcomes. ${ }^{1}$ The significance of ERAS practices stems from both its improvement of the clinical outcome and from its recognizable economic potential. In addition to the ability to offer better perioperative care and a reduced length of hospital stay, ERAS implementation has shown a valuable cost reduction associated with perioperative hospitalization. ${ }^{2,3}$ Nonetheless, ERAS implementation has challenges that should be addressed. One of the most significant challenges during ERAS implementation is variability of drugs used throughout the perioperative period. ${ }^{4}$

Current evidence suggests that patients may exhibit variable responses to standard drugs and doses used, which would render uniform drug administration less than ideal, instead requiring individual adaptation. ${ }^{5,6}$ Guidance for drug
Correspondence: Richard D Urman Department of Anesthesiology, Perioperative and Pain Medicine, Brigham and Women's Hospital, Harvard Medical School, 75 Francis Street, Boston, MA, USA

Tel + I 6177328222

Email rurman@bwh.harvard.edu 
administration is an important component of pre-, intra-, and post-operative ERAS protocols. ${ }^{1,7}$

Every patient has his or her own individual pharmacogenetic profile. ${ }^{8}$ These genetic differences are responsible for each individual's susceptibility to diseases and unique drug response. ${ }^{5,9}$ Drugs rely on enzymes to reach their target, have therapeutic effects, and then are cleared from the body. ${ }^{5}$ Due to the high variability of the genes coding these enzymes, patients may have overactive, normal, or underactive enzymes. ${ }^{6}$ Therefore, a given drug and a given dose are not going to have the same effect in each individual. ${ }^{10}$ Given these variations, drug administration in ERAS protocol should ideally be individualized.

Pharmacogenomic testing has been designed particularly to improve drug administration. ${ }^{10}$ The results of these tests provide guidelines on how to prescribe the optimal drug with the optimal dosage to each patient based on individual's unique genetic profile. ${ }^{10}$ An important consideration of pharmacogenomics testing is the cost effectivity in treatment. According to a review done by Verbelen et al, in over 68 distinct drugs used in a multitude of clinical specialties, more than half of all studies which reached a conclusion for their analyzed drug reported pharmacogenomics impacted treatment to be either cost dominant or cost-effective. ${ }^{11}$ Two genetic variations of interest concerning drug-response variability are SNPs (single-nucleotide polymorphisms) and CNVs (copy-number variations) occurring inside the human genome. ${ }^{10}$ Due to the timeconsuming and resource-demanding nature of mapping an entire genome, modern day pharmacogenomic testing often utilizes "per gene" testing for patient-specific results identifying at-risk predispositions for certain drugs. ${ }^{10}$ TPMT (thiopurine methyltransferase) and CYP2D6 gene tests are among the most commonly utilized today due to their cost-effectiveness and rapid results. ${ }^{10}$ Preemptive testing of several high-risk pharmacogenes is another strategy recently used to obtain patient-specific results before utilizing at-risk drugs with a reputation for patient variability. ${ }^{10}$ Our objective is to review the most recent elements of ERAS practices that involve drug administration and the feasibility of introducing pharmacogenomics testing in ERAS practices to guide drug administration.

\section{Anxiolytics}

Preoperative stress and anxiety negatively affect perioperative outcomes. ${ }^{12-14}$ Establishing a clear strategy to control preoperative anxiety is essential for rapid recovery and early discharge. ${ }^{12}$ Anxiolysis could be achieved via various approaches either pharmacologically or non-pharmacologically. Non-pharmacological approaches rely mainly on providing preoperative patient counseling and education about the surgery and the perioperative plan and management of patient expectations to help reduce anxiety. ${ }^{15,16}$ Although these approaches have the advantage of avoiding premedication-related adverse effects, they require continuous skillful communication and a buildup of patient rapport to successfully relieve anxiety. ${ }^{16}$

On the other hand, the pharmacological approach carries the drawbacks of drug-related adverse effects, inappropriateness of the premedication drugs for some patients due to comorbidities, and the possibility of suboptimum therapeutic effects. From an ERAS standpoint, it is recommended to avoid long-acting anxiolytics $12 \mathrm{hrs}$ before surgery as it may interfere with early mobilization and return of gut function and early feeding with longer hospital stay. ${ }^{17}$ Therefore, anxiolytics are advised to be restricted to the short-acting drugs that would assist in performing regional anesthetic procedures. ${ }^{1,17}$

Benzodiazepines are the most commonly used anxiolytic premedication drugs. The hepatic CYP enzyme family is responsible for metabolizing most benzodiazepines. ${ }^{18}$ Variations and genetic polymorphisms of CYP enzymes have been shown to alter the metabolization and pharmacologic effects of benzodiazepines. ${ }^{19,20}$ For instance, midazolam is metabolized by CYP3A4/5 enzymes. Patients carrying homozygous alleles for CYP3A $5 * 3$ have been noticed to have more than $50 \%$ induction of these enzymes, and thus a higher rate of metabolizing and eliminating midazolam. ${ }^{19}$ However, studies comparing different polymorphisms (CYP3A5*1/*3 and CYP3A5*3/*3) have shown inconsistent variations in midazolam clearance. ${ }^{18}$ Despite the extensive midazolam use as anxiolytic premedication before surgical procedures, inconclusive evidence of clinical variations in polymorphisms of CYP3A5 may require further research to show the efficacy of the pharmacogenomics administration of midazolam. ${ }^{18}$ Table 1 shows examples of commonly used drugs in anesthesia, metabolizing enzyme, known variants, and their impact on metabolism.

\section{Analgesia}

Multimodal analgesia spanning the entire perioperative period is an essential element of ERAS protocols which is used to ultimately lessen postoperative pain. ${ }^{15}$ Nonopioid analgesic use is the adopted strategy for perioperative pain control in most ERAS guidelines. ${ }^{21}$ Nonsteroidal anti-inflammatory drugs (NSAIDs), acetaminophen, and 
Table I Examples of commonly used drugs in anesthesia, corresponding metabolizing enzyme(s), known variants, and their impact on metabolism

\begin{tabular}{|c|c|c|c|}
\hline Drug of interest & Metabolizing enzyme & Variant & Effect \\
\hline I. Midazolam & CYP3A4/5 & CYP3A5*3 & $50 \%$ enzyme induction \\
\hline \multirow[t]{2}{*}{ 2. NSAIDs } & CYP2C8/9 & CYP2C8/9*I & Normal metabolizer \\
\hline & & CYP2C $8 / 9 * 2, * 3$ & Poor metabolizers \\
\hline \multirow[t]{3}{*}{ 3. Ketamine } & Depending on ketamine plasma level: & CYP2B $6 * 6 / * 6$ & Low plasma clearance \\
\hline & - At high levels: CYP3A4 & CYP $2 B 6 * 1 / * 6$ or CYP 2 B $6 * 1 / * 1$ & Better clearance \\
\hline & - At clinically relevant levels: CYP2B6 & & \\
\hline \multirow[t]{2}{*}{ 4. Propofol } & CYP2B6 & CYP2B $6 * 4$ & Reduced clearance \\
\hline & UGTIA9 & & \\
\hline 5. Succinylcholine & $\mathrm{BCHE}$ & BCHE*FSI26, BCHE*I3E4-I4C and BCHE*328D & Prolonged effect \\
\hline \multirow[t]{2}{*}{ 6. Metoclopramide } & CYP2D6 & rs 1080985 and rsI694,7 & Fewer side effects \\
\hline & & rs3892097 & Higher incidence of side effects \\
\hline
\end{tabular}

gabapentinoids (gabapentin, pregabalin) are among the studied drugs that have shown beneficial postoperative results. $^{21-23}$ Additionally, perioperative use of intravenous lidocaine, magnesium, and low-dose ketamine has demonstrated a reduction of postoperative opioid consumption and reported pain scores. ${ }^{24-27}$ These analgesics provide pain control without the adverse effects of their opioid counterparts. $^{22}$ Concerning multimodal analgesia, it is strongly recommended to select a procedure-specific analgesic plan that fits each individual patient. ${ }^{21}$ The availability of a patient's genomic response to drugs used in multimodal opioid-sparing analgesia would allow for optimized drug administration and help to maximize the therapeutic effects while minimizing side effects and reducing the length of hospital stay.

\section{Nonsteroidal anti-inflammatory drugs (NSAIDs)}

NSAIDs inhibit the action of cyclooxygenases (COX-1 and COX-2) on the arachidonic acid which is a cell membrane phospholipid produced during cellular damage. This action prevents prostaglandin production with subsequent decrease of the peripheral nociception and swelling at the time of tissue damage. ${ }^{28}$ NSAIDs include nonselective agents, such as aspirin, ibuprofen, ketorolac, and diclofenac, as well as newer selective COX-2 inhibitors such as parecoxib and celecoxib. Currently, NSAIDs at standard doses are verified as part of the multimodal opioid-sparing analgesic approach by all ERAS Society guidelines. ${ }^{17,21,29-33}$

Some of the recognized drawbacks of NSAIDs include their undesirable effects on the cardiovascular and gastrointestinal systems. ${ }^{34}$ Alternatively, selective COX-2 inhibitors do not show gastrointestinal side effects and thus reduce the risk of peptic ulceration associated with NSAIDs. $^{35}$ However, some COX-2 inhibitors carry an increased cardiovascular risk profile with prolonged use, but this has not been demonstrated with shorter term use. ${ }^{34}$ Understanding the pharmacogenomics of NSAIDs may provide the opportunity of appropriately choosing the suitable drug and its optimum dose.

Most of NSAIDs are metabolized by cytochrome P 450 enzymes (CYP450) - namely CYP2C8 and CYP2C9. ${ }^{36}$ Genetic polymorphisms of these enzymes play an important role in NSAIDs' pharmacokinetics. ${ }^{37}$ Patients with the wild-type CYP2C8*1 or CYP2C9*1 variant show normal metabolism of the drugs. Poor metabolizers carrying the alleles $\mathrm{CYP} 2 \mathrm{C} 8 * 2, * 3, \mathrm{CYP} 2 \mathrm{C} 9 * 2$, or $* 3$ experience abnormally high serum levels due to ineffective drug clearance resulting in unexpected toxicity with standard doses. $^{38,39}$ Therefore, it has been recommended to start treatment at half the normal recommended dose in such patients (CYP2C9*2/*3 genotype) to avoid adverse cardiovascular and gastrointestinal events. ${ }^{37,40}$

\section{Acetaminophen}

Scheduled acetaminophen administration is also recommended by ERAS Society guidelines. ${ }^{17,21,29-33}$ Despite its tolerability and minimal side effect profile, overdose is a risk factor for hepatotoxicity. Additionally, the analgesic potential of NSAIDs and selective COX-2 inhibitors is limited by the degree to which other various endogenous neurotransmitter systems (such as the serotonergic, opioid, and cannabinoid) are inhibited. ${ }^{41}$ Regarding its analgesic potency, it has been shown that analgesia provided by NSAIDs or opioids combined with acetaminophen is superior to analgesia provided by NSAIDs or opioids only. ${ }^{42-45}$ 
Acetaminophen undergoes several processes of extensive hepatic metabolism by multiple enzymes. ${ }^{46}$ These enzymes include UDP glucuronosyltransferases (UGT), cytochrome P (CYP), sulfotransferases (SULT), and glutathione-S-transferases (GST). ${ }^{46}$ Genetic polymorphisms of these enzymes are a major cause of discrepancies in patients' responses to acetaminophen administration. ${ }^{47-51}$ Despite the well-established polymorphism in the four enzymes, studies have been limited to UGT genes only. ${ }^{48,52,53}$ Therefore, studies of genes encoding the other three enzymes are recommended due to their significant contribution in differential drug response. ${ }^{46}$

\section{N-Methyl D-Aspartate receptor antagonists}

Ketamine is one of the well-known N-Methyl D-Aspartate (NMDA) receptor antagonists. ${ }^{22}$ It performs its antagonistic action via noncompetitive inhibition of the excitatory glutamate receptor site on the NMDA channel. ${ }^{22}$ Subanesthetic, intravenous doses of ketamine administered alongside induction agents have been shown to positively manage postoperative pain and reduce opioid consumption. ${ }^{54}$ However, data on ketamine's analgesic role in elective surgeries are limited. ${ }^{22}$ Moreover, side effects such as dizziness and sedation should be considered before its routine use in ERAS guidelines. ${ }^{7,21,55}$ Therefore, pharmacogenomics testing could play a significant role in providing a comprehensive report on patient's genetic profiles and responses to ketamine; thus, ketamine dosages could be optimized and adverse events minimized.

Different types of cytochrome enzymes (CYP2B6, CYP2C9, and CYP3A4) are responsible for metabolizing ketamine via N-demethylation into norketamine. ${ }^{56}$ Depending on ketamine plasma levels, the primary metabolizing isoenzyme usually changes. ${ }^{57}$ For instance, CYP3A4 is the primary metabolizing isoenzyme at higher concentrations of ketamine in plasma, with little contribution from CYP2B6 and CYP2C9. ${ }^{56}$ However, at clinically relevant plasma levels, CYP2B6 takes over the responsibility of metabolizing ketamine, with a little assistance from CYP3A4. Because of this, the metabolization of and clearance of ketamine is highly variable and unpredictable, rendering optimum drug dosage a serious challenge. Additionally, individuals with different variants of CYP2B6 alleles have shown significant differences in ketamine metabolization. ${ }^{58}$ As such, lower plasma clearance, higher plasma levels, and higher incidence of side effects have been noticed in individuals with CYP2B $6 * 6 /$ *6 genotype than those with CYP2B $6 * 1 / * 6$ or CYP2B $6 * 1 /$ $* 1$ genotype. ${ }^{58}$

\section{Opioids}

Optimum intraoperative pain control is mandatory for reduction of surgical stress response and enhancement of recovery. ${ }^{59}$ Opioid use is a cornerstone for pain control in traditional practices. ${ }^{60}$ However, opioid-related side effects are critical barriers for rapid recovery. ${ }^{61}$ Therefore, if deemed essential, short-acting (fentanyl and alfentanil) and ultrashort-acting (remifentanil via infusion) opioids have been recommended over morphine by ERAS Guidelines due to their rapid clearance from the system and fewer side effects. ${ }^{22}$ Due to the variable rate of opioid metabolization and elimination, dosages should be adjusted to each individual patient so as to minimize the incidence of side effects. $^{62}$ A pragmatic study done by UF Health explored the differences in patient responses to opiate medications based on if the individual's pharmacogenomics profile had been considered or not. ${ }^{63}$ They concluded that more extensive research needs to be done on specific medications in order to evaluate the cost-effectiveness and pain control benefits of pharmacogenomics-based opiate treatment. One might argue that testing for opioid metabolism is one example of potentially life-saving pharmacogenomic testing as it can guide short- and long-term therapy and might even prevent an overdose or transition to chronic pain due to inadequate analgesia and opioid use disorder and abuse.

Multiple enzymes are involved in the metabolization of opioids. This provides an opportunity for genetic variability to affect both the efficacy and toxicity of these drugs. Depending on the number of mutant alleles, patients who experience this variability can be classified as ultra-metabolizers with over-functional alleles, extensive-metabolizers with two normal alleles, intermediate-metabolizers with one mutant allele, or poor-metabolizers with two mutant alleles. ${ }^{6}$ CYP2D6 enzyme polymorphism is an example of opioid metabolization variability. There are at least 80 identified CYP2D6 alleles. This allows for wide range of enzymatic variability when compared with the wild-type allele. ${ }^{64}$ Meaningful differences in clinical responses may also occur due to the variable responses of the mutant alleles on a functional gradient. ${ }^{64}$ Therefore, larger studies on this enzyme's polymorphism are required for a more accurate evaluation and a better correlation to clinical responses. ${ }^{36}$

\section{Anesthetics}

There are no studies which compare the different anesthetic agents' used for ERAS to determine evidence-based guidelines. ${ }^{17,21,29,32}$ However, the key goal for anesthetic 
management in ERAS protocols is to facilitate rapid emergence by using the shortest acting agents available. ${ }^{21}$ This has been shown to accelerate recovery and reduce the length of stay, hospital expenses, and postoperative complications. $^{17}$

Depth of anesthesia should be monitored continuously and efficiently to ensure a well-balanced and individualized level of anesthesia to avoid delayed recovery, while still preventing surgical stress and undesired patient awareness. ${ }^{21}$ Bispectral index (BIS) monitoring has been used for monitoring the depth of anesthesia, with BIS levels of 40-60 shown to be sufficient and recommended. ${ }^{21,65}$

Propofol, as one of the commonly used intravenous anesthetic agents, has variable pharmacokinetics attributed to genetic variation of the drug metabolizing enzymes. It undergoes metabolism by hepatic CYP2B6 enzyme and $O$ glucuronidation by UGT1A9. ${ }^{66}$ Polymorphisms of the genes coding for these enzymes affect elimination and clearance of propofol from the system resulting in discrepancies in the serum drug levels. ${ }^{67}$ For instance, patients with CYP2B6*4 alleles have been shown to have reduced drug clearance which increases their susceptibility to drug toxicity even with the regular doses. ${ }^{68}$ In another study, CYP2B6*6 has been demonstrated to be responsible for the differences in plasma drug levels and dose requirements. ${ }^{69}$ Additionally, propofol pharmacodynamics are affected by variations in the genes coding for $\mathrm{GABA}_{\mathrm{A}}$ target receptors (GABRE). ${ }^{70}$ Furthermore, another study suggests that the extensive variability of patient susceptibility could be attributed to a much greater range of genes including the dominant variations in GABAA1 rs2279020, GABAA2 rs11503014, and CHRM2 rs1824024. ${ }^{71}$ Cytochrome P450 family (CYP450), ATP-binding cassette (ABCB1), serine/threonine-protein kinase 3 (TAOK3), family with sequence similarity 53 member $B$ (FAM53B), and the cannabinoid receptor (CNR1) are postulated to be involved in propofol pharmacokinetics; opioid receptors (OPRM1 and OPRD1), $\beta$-adrenoceptor (ADRB1), Catechol-O-methyltransferase (COMT), and ligand-gated ion channel (P2RX7) are postulated to be directly or indirectly involved in the pharmacodynamic response to propofol; nitric oxide synthase (NOS3), GABA type A (GABAA) receptor, NMDA receptors (GR1N3A and GR1N2B), Galanin (GAL), fatty acid amide hydrolase (FAAH), 5-hydroxytryptamine receptor (5HT2A), cholinergic receptors (CHRM2 and CHRNA5), dopamine transporters (DAT and DRD2), casein kinase (CSNK1E), calcium channels, potassium channels
(KCNS1 and GIRK), and sodium channels (SCN9A) are also likely involved in the action of propofol. However, the gene variants so far studied have not shown any significant clinical differences. ${ }^{70}$

\section{Neuromuscular blockade}

For neuromuscular blockade, ERAS Society guidelines have recommended the use of short-acting rather than long-acting neuromuscular blockers. ${ }^{21}$ Monitoring the depth of the blockade with a Train of Four (TOF) nerve stimulator is essential to ensure adequate deep block during surgery and satisfactory reversal of the block at the time of emergence and recovery. ${ }^{21,29}$ A TOF ratio of 0.9 has been shown to be associated with sufficient neuromuscular recovery and increased patient satisfaction at recovery. $^{21,29}$ Additionally, the new reversal agent for neuromuscular blockade, Sugammadex $\AA$, may provide faster reversal than traditional anticholinesterases, with comparable side effects, allowing for quick and safe recovery. $^{21,29,32}$

From a pharmacogenomics standpoint, genetic polymorphism of the butyrylcholinesterase (BCHE) enzyme is one of the most common examples of discrepancies in patients' drug response in the perioperative settings. ${ }^{72}$ $\mathrm{BCHE}$ is responsible for metabolizing succinylcholine in the plasma and liver. ${ }^{73}$ Patients having mutant alleles (BCHE*FS126, BCHE*I3E4-14C, and BCHE*328D) have been shown to experience longer duration of muscle relaxation than patients with wild-type alleles. ${ }^{74}$ Specifically, patients with homozygous mutant alleles are susceptible to more prolonged relaxation than patients with heterozygous alleles. ${ }^{75}$ Thus, variability of genetic expression of this enzyme affects patients' outcomes and could result in longer hospital stay with higher incidence of postoperative morbidity and mortality. ${ }^{76}$

Mei et al demonstrated that the SLCO1B1 and ABCB1 gene variants might affect rocuronium action, and the $\mathrm{ABCB} 1$ rs1128503 $\mathrm{C}>\mathrm{T}$ genotype was the main factor involved in rocuronium effectiveness. ${ }^{77}$

Similarly, malignant hyperthermia is another example of the genetic expression variability which may result in significant differences in patient response to administered drugs in the perioperative settings. ${ }^{78}$ Episodes of malignant hyperthermia may be brought about after the administration of succinylcholine in some patients. $^{76}$ These examples show the importance of pharmacogenomic testing and how significant it is to evaluate patients' genes and test their responses to drugs before administration. ${ }^{76}$ 
Therefore, genetics-guided pharmacotherapy and pharmacogenomics testing should play a central role in ERAS practices to help reduce drug toxicities, optimize patient's drug responses, and thus experience better outcomes and earlier discharge.

\section{Antiemetics}

Postoperative nausea and vomiting (PONV) is a major challenge that should be overcome to ensure an enhanced recovery and shorter length of stay. ${ }^{79}$ Prophylaxis against PONV helps with patient satisfaction and early discharge. ${ }^{80}$ Preoperative antiemetic drugs have been shown to be effective with PONV control..$^{80}$ Use of a combination of medications appears more effective than single drug use. ${ }^{21}$ Patients with medium risk should receive 1-2 prophylactic antiemetic drugs, while high-risk patients should receive $2-3$ drugs. ${ }^{80}$ Non-pharmacologic interventions including: reduction of preoperative fasting, use of carbohydrate-loaded drinks, avoidance of opiates, use of regional anesthetic techniques whenever possible, and propofol-based total intravenous anesthesia also have a valuable contribution to PONV control. ${ }^{21}$

5-hydroxytryptamine type 3 (5-HT3) is the most common target receptor for drugs used for PONV. It has been shown that antagonism of these receptors results in a meaningful reduction of PONV. ${ }^{81}$ Polymorphism of the 5-HT3B receptor gene has been studied and revealed that deletion variants may be a pharmacogenetic predictor for patient responsiveness to ondansetron use for PONV. ${ }^{81}$ Other receptors that may play a role in PONV are dopamine type 2 receptors and mu-opioid receptors. The Dopamine D2 receptor Taq IA polymorphism has been associated with early incidence of PONV. ${ }^{82}$ Additionally, mu-opioids receptors' polymorphisms have been studied and revealed that heterozygous polymorphisms might be related to less opioid consumption with reduced side effects, including opioid-induced PONV, when compared to homozygous polymorphisms. ${ }^{83}$ Sugino et al demonstrated that specific sets of SNP genotypes in the OPRM1 gene might contribute to individual responses to opioid medication and severity of PONV, preoperative genotyping being advised for patients at risk. ${ }^{84}$

\section{Postoperative ileus management}

Postoperative ileus (POI) can be defined as transient episode of gut motility impairment after surgery leading to abdominal distension, vomiting, food intolerance, and late elimination. Besides increased hospitalization costs, this could result in longer hospital stay and increasing susceptibility to its associated morbidities as hospital-acquired infections or venous thromboembolism. ${ }^{85}$ Several approaches have been studied to reduce POI including enhanced recovery protocols..$^{1,7,30,31,55}$ Other recent targeted interventions, such as intravenous lidocaine, chewing gum, and peripheral mu antagonists, have been investigated. However, the clinical evidence for many of these remains controversial. ${ }^{86,87}$

The pharmacological therapeutic strategies for POI included prokinetics and peripheral mu antagonists. Most of the prokinetics used for POI are metabolized by hepatic CYP 450 enzymes system; particularly CYP2D6 for metoclopramide and CYP3A4 for cisapride. ${ }^{88}$ These prokinetics are known for their critical cardiovascular and neurological side effects. ${ }^{89}$ Genetic variations in the metabolic enzymes of these drugs predispose patients to discrepancies in drug efficacy and toxicity. For instance, genetic polymorphisms in the CYP2D6 gene have been shown associated with variability in metoclopramide side effects. Patients with certain polymorphisms, such as rs1080985 and rs16947, have been found to experience fewer side effects than usual while other patients with a different polymorphism such as rs3892097 have shown higher incidence of side effects than expected. ${ }^{90}$ Not only the CYP2D6 genetic variations have shown an association with metoclopramide metabolism and side effects, but KCNH2 and HTR4 genetic polymorphisms also have shown variable tendencies for adverse effects. Additionally, the clinical efficacy of metoclopramide has been associated with polymorphisms in ADRA1D genes. ${ }^{91,92}$ Understanding these genetic variations would help with better managing the perioperative administration of metoclopramide to provide the optimum therapeutic effects, while minimizing side effects and length of hospitalization.

\section{Volatile anesthetics}

There is limited evidence regarding genetic variations and response to volatile anesthetics (VAs), and few populationbased studies have been conducted. It is important to remember that VAs are mostly eliminated through the lungs rather than kidneys and liver. In fact, less than $5 \%$ of currently used VAs are metabolized (sevoflurane, $2 \%$, isoflurane $<1 \%$, desflurane $0.1 \%$ ). ${ }^{19}$ There is also partial elimination by biotransformation mediated by the cytochrome enzyme CYP2E1. ${ }^{93}$ There are known genetic variations of CYP2E1, although there are no studies addressing clinical importance of this variation. Genetic variation has 
been implicated in emergence agitation in response to VAs in children. One study of children undergoing tonsillectomy and adenoidectomy with sevoflurane anesthesia showed that the AA group in the GABRgamma2-nucleotide position 3145 in intron $\mathrm{A} / \mathrm{G}$ had a lower incidence of emergence agitation when compared with the non-AA group. ${ }^{94,95}$ In addition, there are studies suggesting that red heads may have higher VA requirements. One study of female read heads receiving desflurane showed that VA requirement was significantly higher than in dark-haired women, due to a mutation on the melanocortin-1 receptor gene. ${ }^{96}$

\section{Counter arguments}

Pharmacogenomics opened a relatively new and exciting chapter of modern science. However, the FDA warned against the use of pharmacogenetic testing to predict patient-variable responses as the practice has not yet been approved or reviewed by the administration. ${ }^{97}$ This claim is based on insufficient evidence of the proven effectiveness and reliability of pharmacogenomic testing through clinical trials. ${ }^{97}$ While the FDA is not yet advocating or supporting the use of this kind of testing, as more research and clinical trials are completed, definitive answers to questions that have arisen about the reliability of pharmacogenomics will be acquired. Also, the turnaround time (7-10 days up to 4-6 weeks) is an important factor when the test is decided for the patient who needs fast results to prescribe a medication versus the non-urgent preemptive decision. $^{98}$

\section{Future direction}

Improvement of knowledge on ERAS practices is critical to advance the clinical practice and minimize the gap between knowing and doing. ${ }^{99}$ With the current evidences and experiences gained from ERAS practices, it is reasonable to consider ERAS for all surgical procedures and tailoring its practices to suit procedure and patient-specific needs. Now with the evolution of pharmacogenomics, genetics-guided pharmacotherapy, and its impact on clinical outcomes should be thoroughly studied for better understanding and managing drug administration in the settings of ERAS.

\section{Conclusion}

ERAS practices are composed of several elements and many of these involve drug administration. Personalized drug administration in the perioperative settings could play a central role in ERAS practices as it helps reduce drug toxicities and optimize patient's drug responses. ${ }^{100}$ If a certain drug is given, do we know the correct dose? Would genotyping and phenotyping the patient be enough in order to decide on therapy? For new associated drugs, can any drug interaction be preemptively avoided by pharmacogenomic testing? These are the questions awaiting more definitive data from future research initiatives. Therefore, genetics-guided pharmacotherapy and pharmacogenomics testing have great potential to improve patient outcomes, earlier discharge, and lower health care costs matching ERAS objectives.

\section{Acknowledgments}

We acknowledge the contribution (editing the final version of the manuscript) of pre-med students Andrew Costa (The Ohio State University, Columbus, OH, USA) and Garegin Soghomonyan (Claremont McKenna College, Claremont, CA, USA).

\section{Disclosure}

Richard D Urman received research funding from CQuentia Inc. Sergio D Bergese reports personal fees from Baxter, outside the submitted work. The authors report no other conflicts of interest in this work.

\section{References}

1. Dort JC, Farwell DG, Findlay M, et al. Optimal perioperative care in major head and neck cancer surgery with free flap reconstruction: a consensus review and recommendations from the enhanced recovery after surgery society. JAMA Otolaryngol Head Neck Surg. 2017;143:292-303. doi:10.1001/jamaoto.2016.2981

2. Porter ME. What is value in health care? $N$ Engl $J$ Med. 2010;363:2477-2481. doi:10.1056/NEJMp1011024

3. Ljungqvist O, Young-Fadok T, Demartines N. The history of enhanced recovery after surgery and the ERAS society. $J$ Laparoendosc $A d v$ Surg Tech. 2017;27:860-862. doi:10.1089/lap.2017.0350

4. Lyon A, Payne CJ, MacKay GJ. Enhanced recovery programme in colorectal surgery: does one size fit all? World $J$ Gastroenterol. 2012;18:5661. doi:10.3748/wjg.v18.i40.5661

5. Wilkinson GR. Drug metabolism and variability among patients in drug response. $N$ Engl J Med. 2005;352:2211-2221. doi:10.1056/ NEJMra032424

6. CYP2C8 CC, CYP2C19 CD, CYP2E1 CA, CYP3A7 O, OPRK O. A review of the role of genetic testing in pain medicine. Pain Physician. 2014; 17:425-445.

7. Carmichael JC, Keller DS, Baldini G, et al. Clinical practice guidelines for enhanced recovery after colon and rectal surgery from the American Society of Colon and Rectal Surgeons and Society of American Gastrointestinal and Endoscopic Surgeons. Dis Colon Rectum. 2017;60:761-784. doi:10.1097/DCR.0000000000000883

8. Kalow W, Tang B, Endrenyi L. Hypothesis: comparisons of inter- and intra-individual variations can substitute for twin studies in drug research. Pharmacogenetics. 1998;8:283-290.

9. Jaeger M, Stappers MH, Joosten LA, Gyssens IC, Netea MG. Genetic variation in pattern recognition receptors: functional consequences and susceptibility to infectious disease. Future Microbiol. 2015;10:9891008. doi: $10.2217 / \mathrm{fmb} .15 .37$ 
10. Crews KR, Hicks JK, Pui CH, Relling MV, Evans WE. Pharmacogenomics and individualized medicine: translating science into practice. Clin Pharmacol Ther. 2012;92:467-475.

11. Verbelen M, Weale ME, Lewis CM. Cost-effectiveness of pharmacogenetic-guided treatment: are we there yet? Pharmacogenomics J. 2017;17:395. doi:10.1038/tpj.2017.21

12. Kain $\mathrm{ZN}$, Sevarino F, Pincus $\mathrm{S}$, et al. Attenuation of the preoperative stress response with midazolameffects on postoperative outcomes. Anesthesiology. 2000;93:141-147. doi:10.1097/00000542-20000700 0-00024

13. Ljungqvist $\mathrm{O}$, Scott M, Fearon KC. Enhanced recovery after surgery: a review. JAMA Surg. 2017;152:292-298. doi:10.1001/ jamasurg.2016.4952

14. Thompson T, Keogh E, French CC, Davis R. Anxiety sensitivity and pain: generalisability across noxious stimuli. Pain. 2008;134:187-196. doi:10.1016/j.pain.2007.04.018

15. Scott MJ, McEvoy MD, Gordon DB, et al. American Society for Enhanced Recovery (ASER) and perioperative quality initiative (POQI) joint consensus statement on optimal analgesia within an enhanced recovery pathway for colorectal surgery: part 2 - from PACU to the transition home. Perioper Med. 2017;6:7. doi:10.1186/s13741-017-0063-6

16. Kearney M, Jennrich MK, Lyons S, Robinson R, Berger B. Effects of preoperative education on patient outcomes after joint replacement surgery. Orthop Nurs. 2011;30:391-396. doi:10.1097/ NOR.0b013e31823710ea

17. Gustafsson U, Scott M, Schwenk W, et al. Guidelines for perioperative care in elective colonic surgery: Enhanced Recovery After Surgery (ERAS $®)$ Society recommendations. Clin Nutr. 2012;31:783-800. doi:10.1016/j.clnu.2012.08.013

18. Gonsalves SG, Dirksen RT, Sangkuhl K, et al. Clinical Pharmacogenetics Implementation Consortium (CPIC) guideline for the use of potent volatile anesthetic agents and succinylcholine in the context of RYR 1 or CACNA 1S genotypes. Clin Pharmacol Ther. 2019;105:1338-1344. doi:10.1002/cpt.1319

19. Cohen M, Sadhasivam S, Vinks AA. Pharmacogenetics in perioperative medicine. Curr Opin Anesthesiol. 2012;25:419-427. doi:10.1097/ACO.0b013e3283556129

20. Palmer SN, Giesecke NM, Body SC, Shernan SK, Fox AA, Collard CD. Pharmacogenetics of anesthetic and analgesic agents. Anesthesiology. 2005;102:663-671. doi:10.1097/00000542200503000-00028

21. Feldheiser A, Aziz O, Baldini G, et al. Enhanced Recovery After Surgery (ERAS) for gastrointestinal surgery, part 2: consensus statement for anaesthesia practice. Acta Anaesthesiol Scand. 2016;60:289-334. doi:10.1111/aas.12651

22. Beverly A, Kaye AD, Ljungqvist O, Urman RD. Essential elements of multimodal analgesia in enhanced recovery after surgery (ERAS) guidelines. Anesthesiol Clin. 2017;35:e115-e43. doi:10.1016/j.anclin.2017.01.018

23. Hurley RW, Cohen SP, Williams KA, Rowlingson AJ, Wu CL. The analgesic effects of perioperative gabapentin on postoperative pain: a meta-analysis. Reg Anesth Pain Med. 2006;31:237-247. doi:10.1016/j.rapm.2006.01.005

24. Khan JS, Yousuf M, Victor JC, Sharma A, Siddiqui N. An estimation for an appropriate end time for an intraoperative intravenous lidocaine infusion in bowel surgery: a comparative meta-analysis. J Clin Anesth. 2016;28:95-104. doi:10.1016/j.jclinane.2015.07. 007

25. Bao-Lin Guo YL, Wei H, Zhen C-X, et al. Effects of systemic magnesium on post-operative analgesia: is the current evidence strong enough. Pain Physician. 2015;18:405-417.

26. Laskowski K, Stirling A, McKay WP, Lim HJ. A systematic review of intravenous ketamine for postoperative analgesia. Can J Anesth. 2011;58:911. doi:10.1007/s12630-011-9560-0
27. Wang L, Johnston B, Kaushal A, Cheng D, Zhu F, Martin J. Ketamine added to morphine or hydromorphone patient-controlled analgesia for acute postoperative pain in adults: a systematic review and meta-analysis of randomized trials. Can J Anesth. 2016;63:311-325. doi:10.1007/s12630-015-0551-4

28. Kapur BM, Lala PK, Shaw JL. Pharmacogenetics of chronic pain management. Clin Biochem. 2014;47:1169-1187. doi:10.1016/j. clinbiochem.2014.05.065

29. Thorell A, MacCormick A, Awad S, et al. Guidelines for perioperative care in bariatric surgery: enhanced recovery after surgery (ERAS) society recommendations. World J Surg. 2016;40:20652083. doi:10.1007/s00268-016-3492-3

30. Nelson G, Altman A, Nick A, et al. Guidelines for postoperative care in gynecologic/oncology surgery: Enhanced Recovery After Surgery (ERAS $®)$ Society recommendations - part II. Gynecol Oncol. 2016;140:323. doi:10.1016/j.ygyno.2015.12.019

31. Melloul E, Hübner M, Scott M, et al. Guidelines for perioperative care for liver surgery: enhanced recovery after surgery (ERAS) society recommendations. World J Surg. 2016;40:2425-2440. doi:10.1007/s00268-016-3700-1

32. Nygren J, Thacker J, Carli F, et al. Guidelines for perioperative care in elective rectal/pelvic surgery: Enhanced Recovery After Surgery (ERAS®) Society recommendations. Clin Nutr. 2012;31:801-816. doi:10.1016/j.clnu.2012.08.012

33. Lassen K, Coolsen MM, Slim K, et al. Guidelines for perioperative care for pancreaticoduodenectomy: Enhanced Recovery After Surgery (ERAS $®)$ Society recommendations. World J Surg. 2013;37:240-258. doi:10.1007/s00268-012-1771-1

34. Harirforoosh S, Asghar W, Jamali F. Adverse effects of nonsteroidal antiinflammatory drugs: an update of gastrointestinal, cardiovascular and renal complications. $J$ Pharm Pharm Sci. 2014;16:821-847. doi:10.18433/J3VW2F

35. Bjarnason I. Gastrointestinal safety of NSAIDs and over-the-counter analgesics. Int J Clin Pract. 2013;67:37-42. doi:10.1111/ ijcp.2012.67.issue-s178

36. Saba R, Kaye AD, Urman RD. Pharmacogenomics in pain management. Anesthesiol Clin. 2017;35:295-304. doi:10.1016/j. anclin.2017.01.015

37. Ko T-M, Wong C-S, Wu J-Y, Chen Y-T. Pharmacogenomics for personalized pain medicine. Acta Anaesthesiol Taiwan. 2016;54:24-30. doi:10.1016/j.aat.2016.02.001

38. Martínez C, García-Martín E, Blanco G, Gamito FJ, Ladero JM, Agúndez JA. The effect of the cytochrome P450 CYP2C8 polymorphism on the disposition of (R)-ibuprofen enantiomer in healthy subjects. $\mathrm{Br} J$ Clin Pharmacol. 2005;59:62-68. doi:10.1111/j.1365-2125.2004.02183.x

39. Kumar S, Samuel K, Subramanian R, et al. Extrapolation of diclofenac clearance from in vitro microsomal metabolism data: role of acyl glucuronidation and sequential oxidative metabolism of the acyl glucuronide. J Pharmacol Exp Ther. 2002;303:969-978. doi:10.1124/jpet.102.038992

40. Blanco G, Martínez C, Ladero JM, et al. Interaction of CYP2C8 and CYP2C9 genotypes modifies the risk for nonsteroidal antiinflammatory drugs-related acute gastrointestinal bleeding. Pharmacogenet Genomics. 2008;18:37-43. doi:10.1097/FPC.0b0 $13 \mathrm{e} 3282 \mathrm{f} 305 \mathrm{a} 9$

41. Graham GG, Davies MJ, Day RO, Mohamudally A, Scott KF. The modern pharmacology of paracetamol: therapeutic actions, mechanism of action, metabolism, toxicity and recent pharmacological findings. Inflammopharmacology. 2013;21:201-232. doi:10. 1007/s10787-013-0172-x

42. Ong CK, Seymour RA, Lirk P, Merry AF. Combining paracetamol (acetaminophen) with nonsteroidal antiinflammatory drugs: a qualitative systematic review of analgesic efficacy for acute postoperative pain. Anesth Analg. 2010;110:1170-1179. 
43. Ziemann-Gimmel P, Hensel P, Koppman J, Marema R. Multimodal analgesia reduces narcotic requirements and antiemetic rescue medication in laparoscopic Roux-en-Y gastric bypass surgery. Surg Obes Relat Dis. 2013;9:975-980. doi:10.1016/j.soard.2013. 02.003

44. Maund E, McDaid C, Rice S, Wright K, Jenkins B, Woolacott N. Paracetamol and selective and non-selective non-steroidal antiinflammatory drugs for the reduction in morphine-related sideeffects after major surgery: a systematic review. $\mathrm{Br} J$ Anaesth. 2011;106:292-297. doi:10.1093/bja/aeq406

45. Remy C, Marret E, Bonnet F. Effects of acetaminophen on morphine side-effects and consumption after major surgery: meta-analysis of randomized controlled trials. Br J Anaesth. 2005;94:505513. doi:10.1093/bja/aei085

46. Mazaleuskaya LL, Sangkuhl K, Thorn CF, FitzGerald GA, Altman RB, Klein TE. PharmGKB summary: pathways of acetaminophen metabolism at the therapeutic versus toxic doses. Pharmacogenet Genomics. 2015;25:416. doi:10.1097/FPC.0000000000000150

47. Nakagawa T, Mure T, Yusoff S, et al. Acetaminophen administration in a patient with Gilbert's syndrome. Pediatr Int. 2012;54:934 936. doi:10.1111/j.1442-200X.2012.03602.x

48. Tankanitlert J, Morales NP, Howard TA, et al. Effects of combined UDP-glucuronosyltransferase (UGT) 1A1* 28 and 1A6* 2 on paracetamol pharmacokinetics in $\beta$-thalassemia $/ \mathrm{HbE}$. Pharmacology. 2007;79:97-103. doi:10.1159/000097908

49. Buchard A, Eefsen M, Semb S, et al. The role of the glutathione Stransferase genes GSTT1, GSTM1, and GSTP1 in acetaminophenpoisoned patients. Clin Toxicol. 2012;50:27-33. doi:10.3109/ 15563650.2011.639713

50. Nagar S, Walther S, Blanchard RL. Sulfotransferase (SULT) 1A1 polymorphic variants* $1, * 2$, and* 3 are associated with altered enzymatic activity, cellular phenotype, and protein degradation. Mol Pharmacol. 2006;69:2084-2092. doi:10.1124/ mol.105.019240

51. Ueshima Y, Tsutsumi M, Takase S, Matsuda Y, Kawahara H. Acetaminophen metabolism in patients with different cytochrome P-4502E1 genotypes. Alcoholism. 1996;20:25a-8a. doi:10.1111/ acer.1996.20.issue-s1

52. Freytsis M, Wang X, Peter I, et al.; Group ALFS. The UDPglucuronosyltransferase (UGT) 1A polymorphism c. 2042C $>$ G (rs8330) is associated with increased human liver acetaminophen glucuronidation, increased UGT1A exon $5 \mathrm{a} / 5 \mathrm{~b}$ splice variant mRNA ratio, and decreased risk of unintentional acetaminopheninduced acute liver failure. J Pharmacol Exp Ther. 2013;345:297307. doi:10.1124/jpet.112.202010

53. Navarro SL, Chen Y, Li L, et al. UGT1A6 and UGT2B15 polymorphisms and acetaminophen conjugation in response to a randomized, controlled diet of select fruits and vegetables. Drug Metab Dispos. 2011;39:1650-1657. doi:10.1124/dmd.111.039149

54. Siddiqui KM, Khan FA. Effect of preinduction low-dose ketamine bolus on intra operative and immediate postoperative analgesia requirement in day care surgery: a randomized controlled trial. Saudi J Anaesth. 2015;9:422. doi:10.4103/1658-354X.159468

55. Temple-Oberle C, Shea-Budgell MA, Tan M, et al. Consensus review of optimal perioperative care in breast reconstruction: enhanced recovery after surgery (ERAS) society recommendations. Plast Reconstr Surg. 2017;139:1056e-71e. doi:10.1097/PRS.0000 000000003242

56. Hijazi Y, Boulieu R. Contribution of CYP3A4, CYP2B6, and CYP2C9 isoforms toN-demethylation of ketamine in human liver microsomes. Drug Metab Dispos. 2002;30:853-858. doi:10.1124/ dmd.30.7.853

57. Aroke EN, Dungan JR. Pharmacogenetics of anesthesia: an integrative review. Nurs Res. 2016;65:318-330. doi:10.1097/NNR.00 00000000000164
58. Li Y, Jackson KA, Slon B, et al. CYP2B6* 6 allele and age substantially reduce steady-state ketamine clearance in chronic pain patients: impact on adverse effects. $\mathrm{Br} \mathrm{J}$ Clin Pharmacol. 2015;80:276-284. doi:10.1111/bcp.12614

59. McEvoy MD, Scott MJ, Gordon DB, et al. American Society for Enhanced Recovery (ASER) and Perioperative Quality Initiative (POQI) joint consensus statement on optimal analgesia within an enhanced recovery pathway for colorectal surgery: part 1 - from the preoperative period to PACU. Perioper Med. 2017;6:8. doi:10.1186/s13741-017-0064-5

60. Garimella V, Cellini C. Postoperative pain control. Clin Colon Rectal Surg. 2013;26:191-196. doi:10.1055/s-0033-1351138

61. Grant MC, Sommer PM, He C, et al. Preserved analgesia with reduction in opioids through the use of an acute pain protocol in enhanced recovery after surgery for open hepatectomy. Reg Anesth Pain Med. 2017;42:451-457. doi:10.1097/AAP.00000000 00000615

62. Williams D, Patel A, Howard R. Pharmacogenetics of codeine metabolism in an urban population of children and its implications for analgesic reliability. $\mathrm{Br} J$ Anaesth. 2002;89:839-845. doi:10.1093/bja/aef284

63. Smith DM, Weitzel KW, Cavallari LH, Elsey AR, Schmidt SO. Clinical application of pharmacogenetics in pain management. $\mathrm{Per}$ Med. 2018;15:117-126. doi:10.2217/pme-2017-0032

64. Bradford LD. CYP2D6 allele frequency in European Caucasians, Asians, Africans and their descendants. Pharmacogenomics. 2002;3:229-243. doi:10.1517/14622416.3.2.229

65. Punjasawadwong Y, Phongchiewboon A, Bunchungmongkol N. Bispectral index for improving anaesthetic delivery and postoperative recovery. Cochrane Database Syst Rev. 2014. doi:10.1002/ 14651858.CD003843.pub3

66. Mikstacki A, Skrzypczak-Zielinska M, Tamowicz B, ZakerskaBanaszak O, Szalata M, Slomski R. The impact of genetic factors on response to anaesthetics. Adv Med Sci. 2013;58:9-14. doi:10.2478/v10039-012-0065-z

67. Mastrogianni O, Gbandi E, Orphanidis A, et al. Association of the CYP2B6 c. $516 \mathrm{G}>\mathrm{T}$ polymorphism with high blood propofol concentrations in women from northern Greece. Drug Metab Pharmacokinet. 2014;29(2):215-8.

68. Eugene AR. CYP2B6 genotype guided dosing of propofol anesthesia in the elderly based on nonparametric population pharmacokinetic modeling and simulations. Int J Clin Pharmacol Toxicol. 2017;6:242.

69. Mourão AL, de Abreu FG, Fiegenbaum M. Impact of the cytochrome P450 2B6 (CYP2B6) gene polymorphism c. $516 \mathrm{G}>\mathrm{T}$ (rs3745274) on propofol dose variability. Eur J Drug Metab Pharmacokinet. 2016;41:511-515. doi:10.1007/s13318-0150289-y

70. Iohom G, Chonghaile MN, O'brien J, Cunningham A, Fitzgerald D, Shields D. An investigation of potential genetic determinants of propofol requirements and recovery from anaesthesia. Eur J Anaesthesiol. 2007;24:912-919. doi:10.1017/S0265021507000476

71. Zhong Q, Chen X, Zhao Y, Liu R, Yao S. Association of polymorphisms in pharmacogenetic candidate genes with propofol susceptibility. Sci Rep. 2017;7:3343. doi:10.1038/s41598-01703229-3

72. Garcia DF, Oliveira TG, Molfetta GA, et al. Biochemical and genetic analysis of butyrylcholinesterase (BChE) in a family, due to prolonged neuromuscular blockade after the use of succinylcholine. Genet Mol Biol. 2011;34:40-44. doi:10.1590/S1415-4757201 1000100008

73. Delacour H, Lushchekina S, Mabboux I, et al. Characterization of a novel BCHE "silent" allele: point mutation (p. Val204Asp) causes loss of activity and prolonged apnea with suxamethonium. PLoS One. 2014;9:e101552. doi:10.1371/journal.pone.0101552 
74. Gätke MR, Bundgaard JR, Viby-Mogensen J. Two novel mutations in the BCHE gene in patients with prolonged duration of action of mivacurium or succinylcholine during anaesthesia. Pharmacogenet Genomics. 2007;17:995-999. doi:10.1097/FPC.0b013e3282f06646

75. Jensen F, Viby-Mogensen J. Plasma cholinesterase and abnormal reaction to succinylcholine: twenty years' experience with the Danish Cholinesterase Research Unit. Acta Anaesthesiol Scand. 1995;39:150-156.

76. Alvarellos ML, McDonagh EM, Patel S, McLeod HL, Altman RB, Klein TE. PharmGKB summary: succinylcholine pathway, pharmacokinetics/pharmacodynamics. Pharmacogenet Genomics. 2015;25:622. doi:10.1097/FPC.0000000000000170

77. Mei Y, Wang SY, Li Y, et al. Role of SLCO1B1, ABCB1, and CHRNA1 gene polymorphisms on the efficacy of rocuronium in Chinese patients. J Clin Pharmacol. 2015;55:261-268. doi:10.1002/jcph.405

78. Rosenberg H, Pollock N, Schiemann A, Bulger T, Stowell K. Malignant hyperthermia: a review. Orphanet $J$ Rare Dis. 2015;10:93. doi:10.1186/s13023-015-0310-1

79. Habib AS, Gan TJ. Postoperative nausea and vomiting: then \& now. Anesth Analg. 2012;115(3):493-495.

80. Gan TJ, Diemunsch P, Habib AS, et al. Consensus guidelines for the management of postoperative nausea and vomiting. Anesth Analg. 2014;118:85-113. doi:10.1213/ANE.0000000000000002

81. Kim M-S, Lee J-R, Choi E-M, Kim EH, Choi SH. Association of 5-HT3B receptor gene polymorphisms with the efficacy of ondansetron for postoperative nausea and vomiting. Yonsei Med J. 2015;56:1415-1420. doi:10.3349/ymj.2015.56.5.1415

82. Nakagawa M, Kuri M, Kambara N, et al. Dopamine D2 receptor Taq IA polymorphism is associated with postoperative nausea and vomiting. J Anesth. 2008;22:397-403. doi:10.1007/s00540-008-0661-Z

83. Kolesnikov Y, Gabovits B, Levin A, Voiko E, Veske A. Combined catechol-O-methyltransferase and $\mu$-opioid receptor gene polymorphisms affect morphine postoperative analgesia and central side effects. Anesth Analg. 2011;112:448-453. doi:10.1213/ ANE.0b013e318202cc8d

84. Sugino S, Hayase T, Higuchi M, et al. Association of $\mu$-opioid receptor gene (OPRM1) haplotypes with postoperative nausea and vomiting. Exp Brain Res. 2014;232:2627-2635. doi:10.1007/s00221-014-3987-9

85. Iyer S, Saunders WB, Stemkowski S. Economic burden of postoperative ileus associated with colectomy in the United States. J Manag Care Pharm. 2009;15:485-494. doi:10.18553/jmcp.2009.15.6.485

86. Short V, Herbert G, Perry R, et al. Chewing gum for postoperative recovery of gastrointestinal function. Cochrane Database Syst Rev. 2015. doi:10.1002/14651858.CD006506.pub3
87. Kranke P, Jokinen J, Pace NL, et al. Continuous intravenous perioperative lidocaine infusion for postoperative pain and recovery. Cochrane Database Syst Rev. 2015. doi:10.1002/14651858. CD009642.pub2

88. Desta Z, Soukhova N, Mahal SK, Flockhart DA. Interaction of cisapride with the human cytochrome P450 system: metabolism and inhibition studies. Drug Metab Dispos. 2000;28:789-800.

89. Quigley EM. Cisapride: what can we learn from the rise and fall of a prokinetic? J Dig Dis. 2011;12:147-156. doi:10.1111/j.17512980.2011.00491.x

90. Zanger UM, Fischer J, Raimundo S, et al. Comprehensive analysis of the genetic factors determining expression and function of hepatic CYP2D6. Pharmacogenet Genomics. 2001;11:573-585. doi:10.1097/00008571-200110000-00004

91. Ljungqvist O. ERAS - enhanced recovery after surgery: moving evidence-based perioperative care to practice. J Parenter Enteral Nutr. 2014;38:559-566. doi:10.1177/0148607114523451

92. Van der Padt A, van Schaik R, Sonneveld P. Acute dystonic reaction to metoclopramide in patients carrying homozygous cytochrome P450 2D6 genetic polymorphisms. Neth $\mathrm{J}$ Med. 2006;64:160-162.

93. Yasny JS, White J. Environmental implications of anesthetic gases. Anesth Prog. 2012;59:154-158. doi:10.2344/0003-3006-59.4.154

94. Kim JH. Mechanism of emergence agitation induced by sevoflurane anesthesia. Korean J Anesthesiol. 2011;60:73-74. doi:10.4097/ kjae.2011.60.2.73

95. Nair AS. Pharmacogenomics of inhalational anesthetic agents. Med Gas Res. 2019;9:52-53. doi:10.4103/2045-9912.254641

96. Liem EB, Lin CM, Suleman MI, et al. Anesthetic requirement is increased in redheads. Anesthesiology. 2004;101:279-283. doi:10.1097/00000542-200408000-00006

97. DRH's Division of Industry Communication and Education (DICE). The FDA Warns against the Use of Many Genetic Tests with Unapproved Claims to Predict Patient Response to Specific Medications: FDA Safety Communication. Silver Spring, MD; 2018.

98. Moyer AM, Vitek CRR, Giri J, Caraballo PJ. Challenges in ordering and interpreting pharmacogenomic tests in clinical practice. $\mathrm{Am}$ J Med. 2017;130:1342-1344. doi:10.1016/j.amjmed.2017.07.012

99. Kehlet H. Enhanced Recovery After Surgery (ERAS): good for now, but what about the future? Can J Anesth. 2015;62:99-104. doi:10.1007/s12630-014-0261-3

100. Saba R, Kaye AD, Urman RD. Pharmacogenomics in anesthesia. Anesthesiol Clin. 2017;35:285-294. doi:10.1016/j.anclin.2017.01.014
Pharmacogenomics and Personalized Medicine

\section{Publish your work in this journal}

Pharmacogenomics and Personalized Medicine is an international, peer-reviewed, open access journal characterizing the influence of genotype on pharmacology leading to the development of personalized treatment programs and individualized drug selection for improved safety, efficacy and sustainability. This journal is indexed on the American Chemical Society's Chemical Abstracts Service (CAS). The manuscript management system is completely online and includes a very quick and fair peer-review system, which is all easy to use. Visit http://www.dovepress.com/testimonials.php to read real quotes from published authors. 